\title{
Factores que influyen en el manejo de los residuos sólidos municipales, Pomacochas, Amazonas
}

\section{Factors that influence the management of municipal solid waste, Pomacochas, Amazonas}

\author{
Rolando Salas López ${ }^{1 *}$, Handerson Mau Goñas Pinedo y Ercy Jhuly Sanchez Sorue
}

\section{RESUMEN}

Los residuos sólidos constituyen un gran problema en nuestro país, debido al desmesurado crecimiento demográfico y los cambios de hábitos de la población orientados al consumo de productos desechables. En este sentido, esta investigación se realizó con el fin de evaluar los factores que influyen en el almacenamiento, recolección, transporte y disposición final de los residuos sólidos municipales en la localidad de Pomacochas. Para ello, se realizó una encuesta para recolectar la información necesaria que permitió identificar los diversos factores influyentes en las etapas de estudio. Esta encuesta fue aplicada en la zona urbana teniendo en cuenta el plano catastral. La muestra fue de 136 encuestas aplicadas mediante muestreo aleatorio. Los resultados obtenidos en la variable residuos sólidos muestran que no se cumple con los lineamientos dados por la Ley General de Residuos Sólidos (Ley N²7314) y por el MINAM. Además los factores sociales y políticos son los que influyen en el manejo de los residuos, en virtud de la escasa participación de la población, ya que no existen programas de separación, que ayuden a minimizar el impacto negativo de los residuos. Además, no se realizan capacitaciones en el manejo de residuos sólidos a pesar de ser una herramienta fundamental para generar conciencia ambiental en la población. Por lo tanto, es necesario implementar un plan operativo para la mejora del sistema de manejo de los residuos sólidos con el fin de brindar un servicio de calidad y la seguridad sanitaria de la población.

Palabras claves: Residuos sólidos, etapas, factores, municipalidad, educación.

\begin{abstract}
Solid waste is a big problem in our country, due to the disproportionate population growth and changes in the habits of the population oriented to the consumption of disposable products. In this sense, this research was carried out in order to evaluate the factors that influence the storage, collection, transport and final disposal of municipal solid waste in the town of Pomacochas. To do this, a survey was conducted to collect the necessary information that allowed identifying the various influential factors in the study stages. This survey was applied in the urban area taking into account the cadastral plan. The sample was 136 surveys applied by random sampling. The results obtained in the variable solid waste show that the guidelines given by the General Law of Solid Waste (Law $\mathrm{N}^{\circ}$ 27314) and MINAM are not complied with, as well as social and environmental factors. Politicians are those who influence the management of waste, by virtue of the scarce participation of the population because there are no separation programs, which help to minimize the negative impact of waste. In addition, there is no training in the management of solid waste despite being a fundamental tool to generate environmental awareness in the population. Therefore, it is necessary to implement an operational plan to improve the solid waste management system in order to provide a quality service and ensure the health of the population.
\end{abstract}

Keywords: Solid waste, stages, factors, municipality, education.

\footnotetext{
Universidad Nacional Toribio Rodríguez de Mendoza de Amazonas (UNTRM-A), Instituto de Investigación para el Desarrollo Sustentable de Ceja de Selva, Calle Higos Urco N 342-350-356, Calle Universitaria Nº304, Chachapoyas, Perú

"Autor de correspondencia. E-mail: rsalas@indes-ces.edu.pe
} 


\section{INTRODUCCIÓN}

Entre los residuos sólidos se incluyen residuos peligrosos y no peligrosos, ambos según definición del Resource Consevation and Recovery Act de la Agencia de los Estados Unidos para el Medio Ambiente (USEPA). Si bien existe un mayor nivel de conciencia tanto en las autoridades como en la sociedad en general sobre los impactos negativos del mal manejo de los residuos sólidos, y por ende una mayor decisión política para mejorar la situación en el sector, los problemas que contribuyen a un manejo deficiente de los residuos sólidos en la región siguen siendo los mismos desde hace 30 años (Terraza, 2009).

Los residuos sólidos constituyen desde hace mucho tiempo un gran problema en nuestro país. Es el caso de los residuos sólidos urbanos, una de las causas principales es la generación por parte de los vecinos, que se preocupan solamente en deshacerse de sus residuos, sin prestar atención al destino que les espera y las consecuencias que acarreará al ambiente y la salud de la población (Municipalidad Distrital de Jazán, 2016).

El desmesurado crecimiento demográfico y los cambios de hábitos de la población, orientados al consumo de productos desechables, así como el inadecuado manejo y disposición final de los residuos sólidos, ha traído consigo muchos problemas de contaminación y enfermedades para las poblaciones aledañas (Vargas, 2015). El distrito de Florida, no es ajeno a esta problemática, y es consciente del reto que significa trabajar en forma integral y adecuada en la gestión de los residuos sólidos domiciliarios que se generan en la localidad de Pomacochas.

Es por estas razones que se ha motivado dedicar esfuerzos para elaborar la presente investigación, la cual permitirá determinar los factores que influyen en el almacenamiento, recolección, transporte y disposición final adecuada de los residuos sólidos municipales en la localidad de Pomacochas, y de esta manera ayudar en la mejora del manejo integral, sostenible y participativo de los residuos generados, mediante un proceso articulado de actividades que ejecuten los actores del área urbana.

\section{MATERIALES Y MÉTODOS}

El presente estudio se realizó en el distrito de FloridaPomacochas, en la provincia de Bongará, Región Amazonas (Figura 1). Para obtener la información requerida se hizo uso de encuestas aplicadas a través de un cuestionario bajo la forma de entrevistas individuales efectuadas a personas según la muestra calcula-

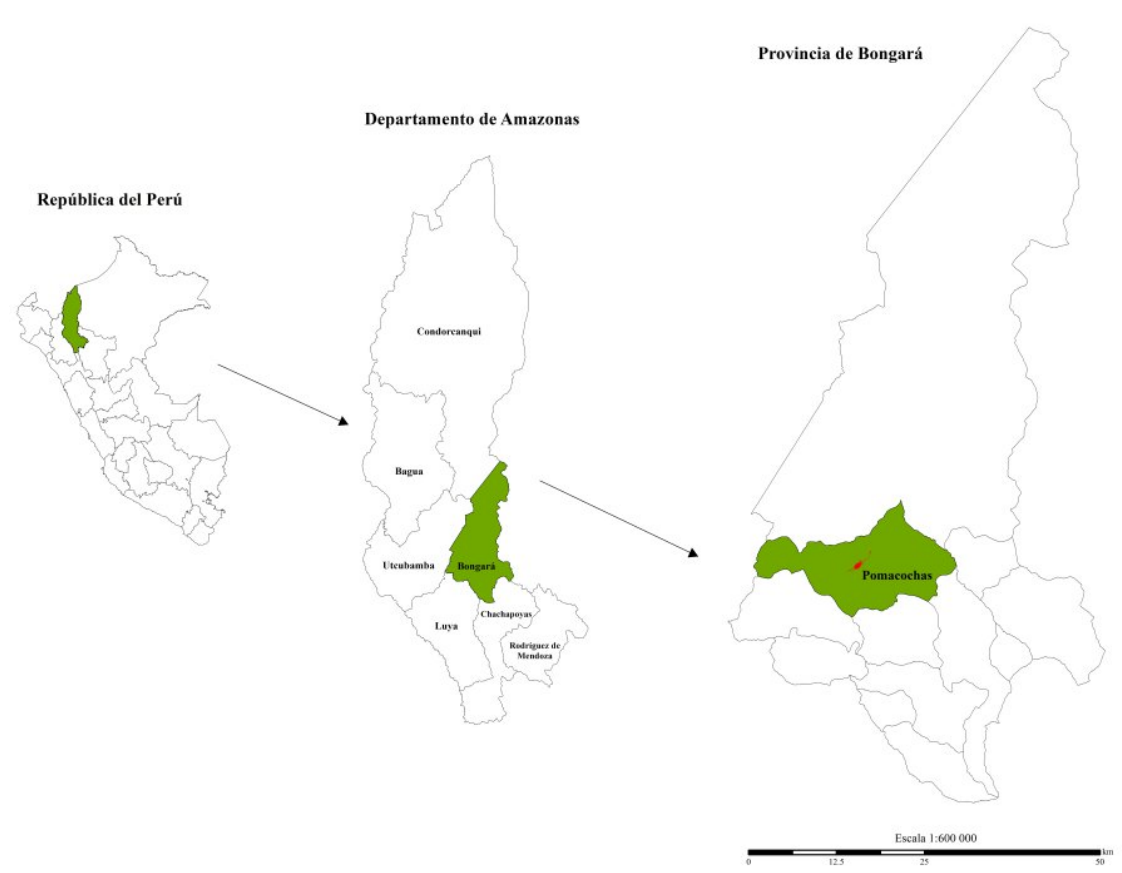

Figura 1. Mapa de ubicación del sector INIA en la ciudad de Chachapoyas 
da. Para obtener esta muestra se optó por aplicar el muestreo irrestricto aleatorio. Se formuló la encuesta definitiva, estructurada con preguntas que buscan identificar los factores sociales, ambientales y políticos en el manejo de los residuos sólidos municipales. El tamaño de la muestra (136 viviendas) fue calculado a partir del total de viviendas (1137 viviendas), utilizando la fórmula establecida por MINAM (2000).

Cabe señalar que para la construcción de las figuras se realizó el análisis porcentual basado en una distribución de frecuencias, donde el valor más frecuente coincide con una tendencia central llamada moda, lo cual permitió la construcción de representaciones gráficas. Esto facilitó la comprensión de los resultados a través de estadísticos descriptivos
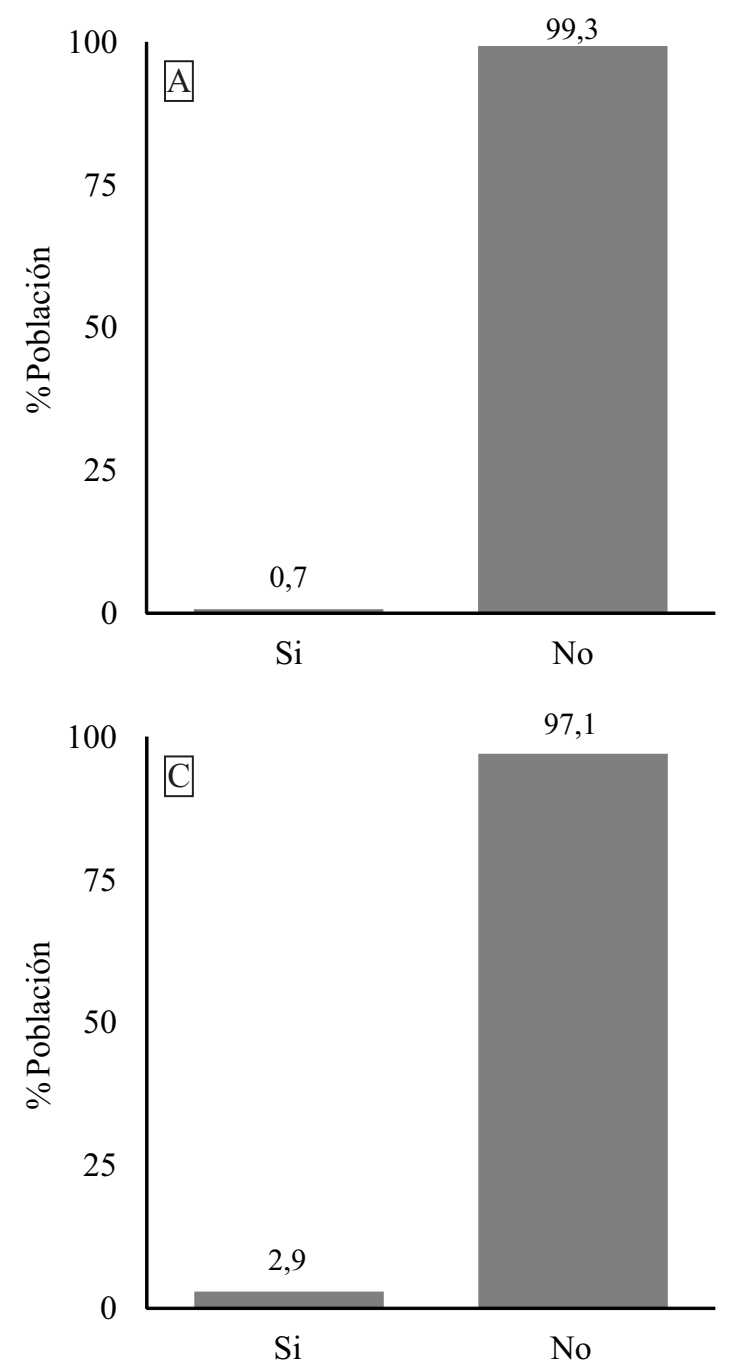

\section{RESULTADOS Y DISCUSIÓN}

Factor Social

\section{Participa en programa de separación}

La mayor parte de la población manifiesta que no participa en ningún programa de separación de residuos. Esto se puede evidenciar en el análisis porcentual que indica un 99,3\% de informantes que respondieron "no". Además se puede apreciar que el 0,7\% si participa (Figura 2A)..

\section{Capacitación}

En el análisis porcentual se evidencia que el 97,1\% de la población no ha recibido capacitación durante el último año. Así mismo se puede evidenciar que un 2,9\% si recibió capacitación (Figura 2C). Esto es debido a que en la localidad no se vienen realizando accio-
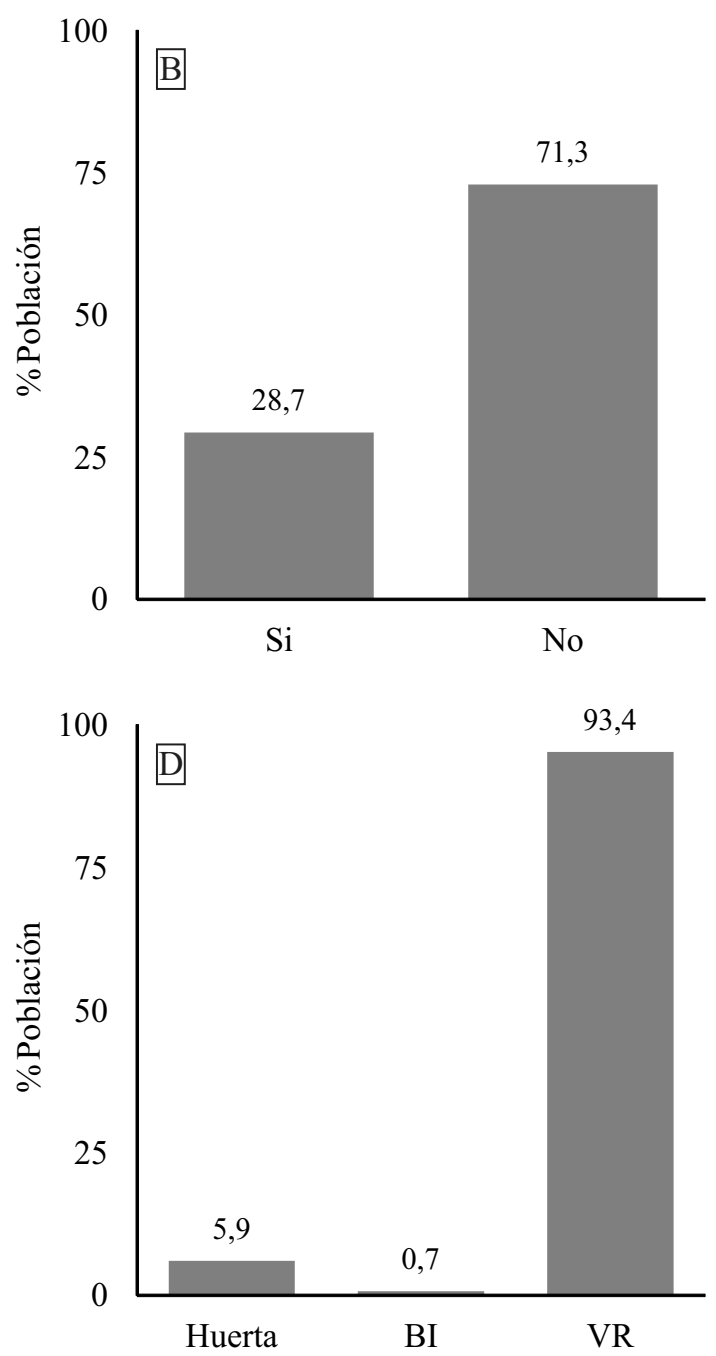

Figura 2. Estadísticos descriptivos derivados de las encuestas realizadas en el distrito de Florida-Pomacochas. Porcentaje de participación en programas de segregación de residuos (A); Porcentaje de personas que conocen los beneficios de un medio ambiente saludable; Porcentaje de personas que recibió capacitación el último año (C); Forma en que las personas disponen su residuos (D). BI: Botadero Informal; VR: Vehiculo Recolector. 
nes para mejorar la educación de la población en el manejo de los residuos sólidos.

Estos resultados evidencian una deficiente participación social en el manejo de residuos en virtud de la inexistencia de programas de separación que ayuden a minimizar residuos inorgánicos. Además no se realizan capacitaciones en residuos sólidos a pesar de ser una herramienta fundamental para generar conciencia ambiental en la población.

\section{Factor Ambiental}

\section{Beneficios del medio ambiente}

La población indica que no tiene conocimiento de los beneficios que brinda el medio ambiente, tal como se evidencia en el análisis porcentual, que indica que un $71,3 \%$ de encuestados respondió con "no" a esta pre-
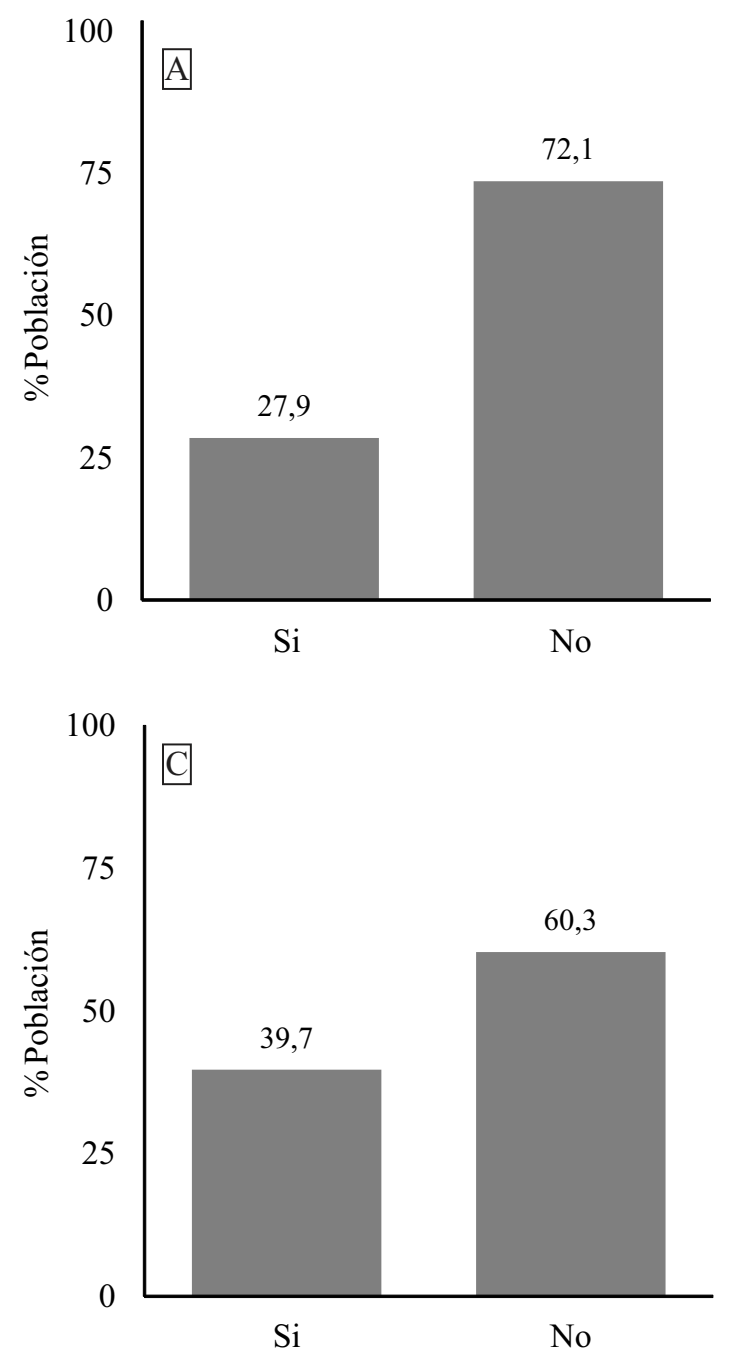

gunta. Sin embargo, el análisis permite evidenciar que un $28,7 \%$ reconoce conocer los beneficios que brinda al medio ambiente (Figura 2B).

\section{Disposición de residuos sólidos}

La población predominantemente dispone sus residuos sólidos generados en su vivienda al vehículo recolector, tal como evidencia el análisis porcentual con un 93,4\% (Figura 2D), debido a que en el lugar se cuenta con el servicio de recolección de residuos que brinda la Municipalidad Distrital. Otros métodos de eliminación menos importantes son "botadero informal" o "en sus huertas" donde los residuos orgánicos formarán parte del suelo.

\section{Impacto ambiental}

En la Figura 3C se evidencia que un $60,3 \%$ de la pobla-
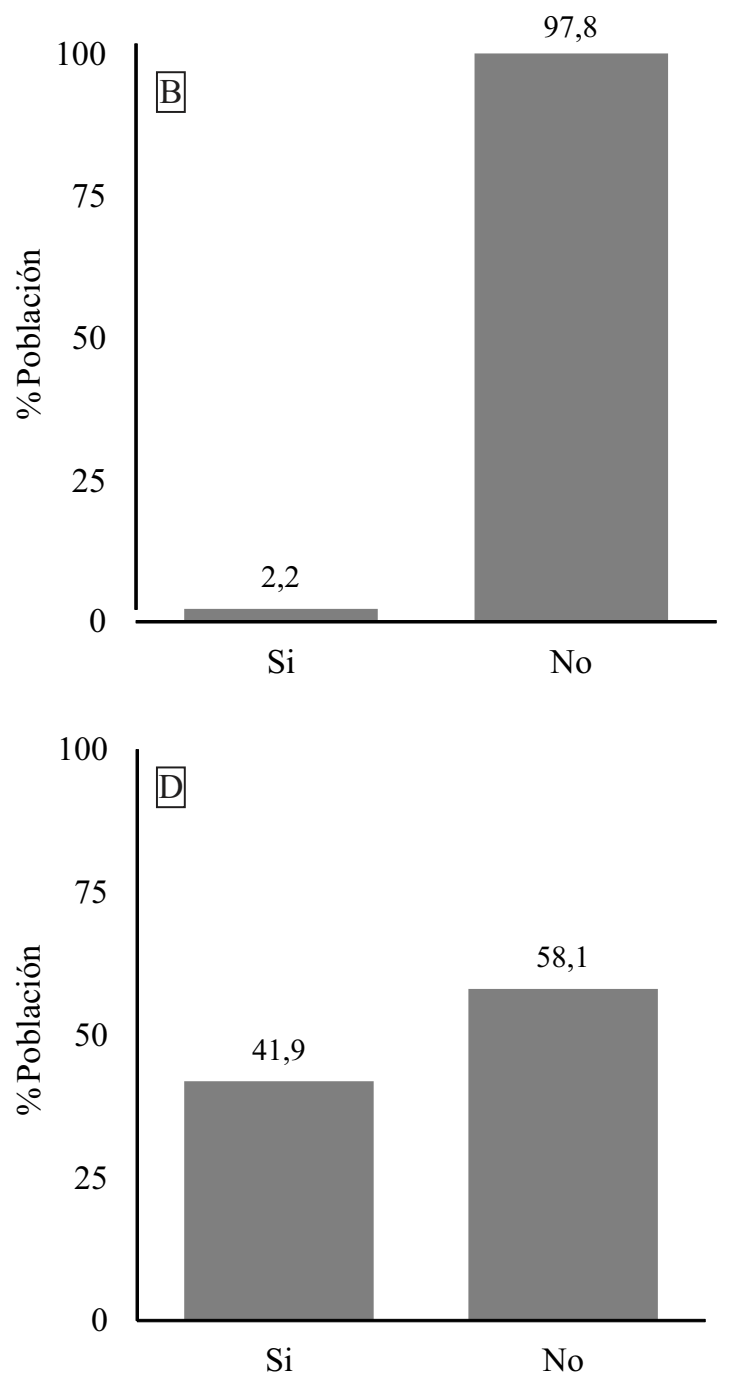

Figura 3. Estadísticos descriptivos derivados de las encuestas realizadas en el distrito de Florida-Pomacochas. Porcentaje de personas que considera que la municipalidad tiene como prioridad el manejo de residuos (A); Porcentaje de personas que conoce de la existencia de un programa de residuos (B); Porcentaje de personas que conoce el impacto de la mala disposición de residuos $(\mathrm{C})$; Porcentaje de personas que se sienten satisfechas con el servicio de recolección de residuos(D). 
ción manifiesta que desconoce el impacto ambiental de una mala disposición de residuos sólidos. Sin embargo, en el análisis también se muestra que el $39,7 \%$ tiene conocimiento respecto a este tema. La razón principal es debido a que la población, en su formación académica, no fue instruida en esta temática. Además no está comprometida en el cuidado del medio ambiente, aduciendo que no es su responsabilidad.

De acuerdo a los resultados se califica el factor ambiental como aceptable en virtud de la forma como disponen sus residuos sólidos entregando al vehículo recolector. Sin embargo, es preciso mencionar que hay una deficiencia de información respecto al beneficio que brinda el medio ambiente saludable y el impacto ambiental de una mala disposición. Finalmente se evidencia una vez más, que es necesario comprometer a la población en el cuidado del medio ambiente.

\section{Factor Político}

\section{Programa de separación de la municipalidad}

La mayor parte de la población manifiesta que la Municipalidad no cuenta con un programa de separación de residuos sólidos tal como se puede evidenciar en el análisis porcentual que arroja un 97,8\% de informantes en esta línea (Figura 3B).

\section{Prioridad de municipalidad}

De acuerdo a los resultados se evidencia que el 72,1\% de la población manifiesta que no considera los residuos sólidos una prioridad dentro del plan de trabajo municipal. Por el contrario, del análisis se puede evidenciar que el $27,9 \%$ considera que sí es prioridad para la Municipalidad el manejo de residuos (Figura 3A). La razón del porcentaje elevado de sensación de desinterés por parte de la Municipalidad es debido a que la población indica que no observa mejoras en el servicio.

\section{Satisfacción por el servicio}

En su mayoría la población indica que no se encuentra satisfecha con el servicio de recolección, como se evidencia en el análisis porcentual, arrojando un $58,1 \%$. Sin embargo, el análisis permite identificar que el $41,9 \%$ si se encuentra satisfecho (Figura 3D). La razón por la cual no están satisfechos es debido a que el servicio no se brinda a toda la población. Esto se observa en la presencia de calles inaccesibles donde el vehículo es inadecuado y la frecuencia es insuficiente. Al respecto los resultados dan indicios de medidas políticas deficientes o inadecuadas en virtud de la inexistencia de programa de separación de residuos. No es prioridad dentro del plan de trabajo municipal, falta voluntad por mejorar el servicio, y la población en su mayoría se encuentran insatisfecha con el servicio y con la falta de compromiso que muestra la autoridad competente ante el problema del manejo de los residuos sólidos.

\section{Consideraciones}

Los resultados en cuanto a la influencia de los factores sociales y ambientales, evidenciaron que para realizar un manejo eficiente de los residuos sólidos municipales, es necesario involucrar a la población y educar en temas ambientales para que se sientan parte de esta problemática y contribuyan al cuidado del medio ambiente. Es así que hasta un 99,3\% no separa los residuos sólidos y un $97,1 \%$ no recibe capacitación, ocasionando como consecuencia que el $60,3 \%$ de la población desconozca sobre los impactos ambientales de una mala disposición de los residuos sólidos. Esto se asemeja a la afirmación de Gouveia (2012) en la que señala que la gestión de residuos sólidos urbanos son fundamentalmente decisiones sobre salud pública y requieren, por lo tanto, la integración entre políticas económicas, sociales y ambientales. Además Pichardo (2009) indica que los problemas que se originan respecto a los residuos sólidos se aliviarían sustancialmente si la población tuviera acceso a una educación profunda sobre la naturaleza, el medio ambiente y el desarrollo sostenible. Estos estudios permiten afirmar que la localidad de Pomacochas no es ajena a la falta de educación ambiental originando problemas en el manejo de los residuos.

También es preciso mencionar que la influencia del factor político juega un papel importante en el manejo de los residuos sólidos. Esto se evidencia en un 72,1\% de la población, quienes afirman que los residuos 
sólidos no están dentro del plan de trabajo de la Municipalidad, además de por la falta de recursos económicos con los que cuenta la Municipalidad Distrital. Por lo tanto, un $58,1 \%$ de la población no está satisfecha con el servicio de recolección de los residuos que se brinda. Cabe destacar que, actualmente, según la legislación vigente, las municipalidades debe proveer el servicio de limpieza pública. Esto se puede corroborar con la afirmación de Castañeda y Pérez (2015), de que uno de los problemas más graves que enfrentan es la falta de recursos económicos para realizar tales funciones.

\section{CONCLUSIONES}

En el manejo de los residuos sólidos municipales, los factores influyentes son el social y el político, puesto que existe desconocimiento por parte de la población en el manejo adecuado de los residuos sólidos municipales y el desinterés de la autoridad local frente a esta problemática y la falta de proposición técnica para mejorar el sistema.

La influencia del factor ambiental se ve reflejada en la falta de información a la comunidad respecto al sitio de disposición final, lo que origina que la población desconozca dicho lugar con la consecuente apertura de botaderos informales, y por consiguiente, la generación de contaminación ambiental afectando la salud de la población cercana.

\section{REFERENCIAS BIBLIOGRÁFICAS}

Castañeda G. A. y A.A. Pérez. 2015. "La problemática del manejo de los residuos sólidos en seis municipios del sur de Zacatecas". Región y sociedad 27:97-115.

Gouveia N. 2012. "Resíduos sólidos urbanos: impactos socioambientais e perspectiva de manejo sustentável com inclusão social". Ciência \& Saúde Coletiva 17: 1503-1510.

MINAM - Ministerio del Ambiente. 2000. Guía metodológica para el desarrollo del Plan de Manejo de Residuos Sólidos. Lima (Perú): M I N A M. R e c u p e r a d o de:http://www.indeci.gob.pe/norma_leg/nor _con/ley_n27314_ley_gen_res_sol.pdf

Municipalidad Distrital de Jazán. 2016. Plan de Manejo de Residuos Sólidos Municipales del Distrito de Jazán, Provincia de Bongará. Jazán (Perú): Sin Editorial.

Pichardo I. 2009. "Responsabilidades municipales en materia ambiental". Convergencia 16: 291308.

Terraza, H. 2009. Lineamientos estratégicos del Banco Interamericano de Desarrollo para el sector de residuos sólidos. Washington (USA): Banco Interamericano de Desarrollo.

Vargas, S. 2015. Factores socioeconómicos que influyen en la inadecuada gestión integral de los residuos sólidos en el Distrito de María, Provincia de Luya, Departamento de Amazonas- 2015. Tesis de grado. Universidad Nacional Toribio Rodríguez de Mendoza de Amazonas. Chachapoyas (Perú). 